\title{
Aqueous Gel-Permeation Chromatography of Electrolytes and Polyelectrolytes I. Effect of Electrolytic Nature of Gel
}

\author{
Satoru KADOKURA, Takeaki MiYAmoto, and Hiroshi InAGAKI \\ Institute for Chemical Research, Kyoto University, \\ Uji, Kyoto 611, Japan
}

(Received August 6, 1982)

\begin{abstract}
This work deals with the effects of charges carried by the stationary phase in aqueous GPC on the elution behavior of simple electrolytes. Simple salts such as $\mathrm{NaCl}, \mathrm{CaCl}_{2}$ and $\mathrm{AlCl}_{3}$ were eluted from a Sephadex gel column using the equilibrium saturation method developed by Sebille et al. It was found that the conventional GPC procedure gives anomalous chromatograms, i.e., appreciably skewed ones, depending on the sample salt concentrations, $C_{\mathrm{s}}$, while an accurate relation between the elution volume, $V_{\mathrm{e}}$, and $C_{\mathrm{s}}$ can be obtained by the equilibrium saturation method. The $V_{\mathrm{e}}$ for the sample salt decreased with increasing $C_{\mathrm{s}}$ and, at a given $C_{\mathrm{s}}$, increased with increasing valency of the salt cation. These results were compared with a theory formulated on the basis of the Donnan equilibrium between the inside and outside of the gel phase carrying fixed charges. It was shown that the anomalous elution behavior of electrolytes can be quantitatively interpreted in terms of the Donnan salt exclusion effect.
\end{abstract}

KEY WORDS Aqueous GPC / Equilibrium Saturation Method / Donnan

Salt Exclusion / Donnan Equilibrium / Electrolyte / Polyelectrolyte /

Elution behavior of simple electrolytes and polyelectrolytes in aqueous gel-permeation chromatography (GPC) has been studied in recent years. $^{1-8}$ The chromatograms obtained exhibit three types of electrostatic effects, depending on the ionic strength of the eluent. One effect arises from an electrostatic interaction between the ionic solute and the background salt in the eluent and can be attributed to a Donnan salt exclusion on the gel. ${ }^{8}$ Another effect is due to an electrostatic repulsion between fixed charges on a polyelectrolyte, which varies with the ionic strength of the eluent. ${ }^{4,5,7}$ These two effects are independent of the electrolytic nature of the gel. Many of the gels available for aqueous GPC have some fixed charges within the gel matrix. ${ }^{1,4,7}$ When such gels are used, a third effect is frequently observed. ${ }^{1,4,7}$ When a sample electrolyte at low concentration is eluted on a column, which contains gels having fixed charges, with pure water or an aqueous salt solution of low ionic strength, the sample ions are excluded from the gel and the sample peak emerges at or near the void vol- ume. Further increase in sample concentration shifts the peak position of the elution curve to a higher elution volume, and, in addition, the chromatogram exhibits a diffuse front side and a sharp back side, which makes it extraordinarily skewed. This effect is considered to arise from the electrostatic repulsion between the ionic solutes and the fixed charges in the gel. However, no quantitative study on this effect has as yet been reported for the following reason. In addition to the unusual skewing in the chromatogram, as far as the conventional GPC procedure has been applied to the experiments, the sample concentration in the column is not the same as the initial sample concentration because of a decrease in the sample concentration due to axial dispersion during the elution. It is impossible to make a quantitative analysis of above-mentioned effect from the chromatogram obtained.

Recently, we found that the equilibrium saturation method, developed by Sebille et al. ${ }^{9}$ for studying the interaction of small ligand molecules with macromolecules, was applicable to a quanti- 
tative study on the third effect mentioned above. This method involves the following three processes: (i) equilibration of a size exclusion column with a solution containing a desired mixture of a small molecule and a macromolecule, (ii) injection of a small volume of the solvent alone, i.e., a solution free of both small and macromolecules, into the column, and (iii) elution of the column with the same solution used for the equilibration. During the elution, two negative peaks related to the concentration of the small molecule appear in the chromatogram. The first negative peak, which emerged at the void volume of the column, reflects the deficiency of small bound molecules, and the second negative peak, the deficiency of small free molecules. In the present study, we modified this method as follows. The column was equilibrated with an aqueous solution containing a simple salt of known concentration. A small amount of pure water was injected into this column as if it were the sample, and then the elution was carried out with the same solution used for the equilibration. As a consequence, only one negative peak appeared in the chromatogram, and the elution volume for this peak varied with the salt concentration in the eluent.

In this paper, the elution behavior of simple salts such as $\mathrm{NaCl}, \mathrm{CaCl}_{2}, \mathrm{AlCl}_{3}$ from a Sephadex G-25 column was investigated by the equilibrium saturation method modified as mentioned above. The results obtained were successfully analyzed according to a theory based on the Donnan equilibrium.

\section{THEORETICAL}

Consider a conventional aqueous GPC column filled with gel particles carrying a small number of fixed anionic charges and exerting no size exclusion effect on any mobile ion species. Further, assume that the fixed anionic charges are uniformly distributed throughout the gel phase.

When such a system is saturated with an aqueous solution of a $1: 1$ simple salt $\mathrm{K}^{+} \mathrm{A}^{-}$, the equilibrium of the system may be determined by the equality of the electrochemical potentials of the two mobile ions, $\mathrm{K}^{+}$and $\mathrm{A}^{-} .{ }^{10}$ Thus,

$$
\left(\gamma_{ \pm}{ }^{0}\right)^{2} C_{\mathbf{K}}{ }^{o} C_{\mathbf{A}}{ }^{o}=\left(\gamma_{ \pm}{ }^{i}\right)^{2} C_{\mathbf{K}}{ }^{i} C_{\mathrm{A}}{ }^{\mathrm{i}}
$$

where $\gamma_{ \pm}$is the mean molar ionic activity coefficient, $C_{\mathrm{K}}$ and $C_{\mathrm{A}}$ are the concentration of the cation $\mathrm{K}^{+}$ and anion $\mathrm{A}^{-}$, respectively, and the superscripts $\mathrm{O}$ and i refer to the outside and inside of the gel phase, respectively. On the other hand, the condition of electrical neutrality gives

$$
C_{\mathrm{K}}{ }^{\circ}=C_{\mathrm{A}}{ }^{\circ}=C_{\mathrm{s}}{ }^{\circ}
$$

and

$$
C_{\mathrm{K}}{ }^{\mathrm{i}}=C_{\mathrm{A}}{ }^{\mathrm{i}}+C_{\mathrm{g}}=C_{\mathrm{s}}{ }^{\mathrm{i}}+C_{\mathrm{g}}
$$

where $C_{\mathrm{s}}$ and $C_{\mathrm{g}}$ are the concentration of the salt and the anionic charges fixed in the gel phase, respectively. Combination of these conditions with eq 1 leads to

$$
R\left(C_{\mathrm{A}}{ }^{\mathrm{o}}\right)^{2}=\left(C_{\mathrm{A}}^{\mathrm{i}}\right)^{2}+\left(C_{\mathrm{A}}^{\mathrm{i}}\right)\left(C_{\mathrm{g}}\right)
$$

and

$$
R\left(C_{\mathrm{K}}{ }^{\mathrm{o}}\right)^{2}=\left(C_{\mathrm{K}}^{\mathrm{i}}\right)^{2}-\left(C_{\mathrm{K}}^{\mathrm{i}}\right)\left(C_{\mathrm{g}}\right)
$$

where

$$
R=\left(\gamma_{ \pm} \mathrm{o} / \gamma_{ \pm}^{\mathrm{i}}\right)^{2}
$$

Equations 4 and 5 may be rewritten

$$
R=\left(C_{\mathrm{A}}{ }^{\mathrm{i}} / C_{\mathrm{A}}{ }^{0}\right)^{2}+\left(C_{\mathrm{A}}{ }^{\mathrm{i}} / C_{\mathrm{A}}{ }^{0}\right)\left(C_{\mathrm{g}} / C_{\mathrm{s}}{ }^{0}\right)
$$

and

$$
R=\left(C_{\mathrm{K}}^{\mathrm{i}} / C_{\mathrm{K}}\right)^{2}-\left(C_{\mathrm{K}}^{\mathrm{i}} / C_{\mathrm{K}}{ }^{0}\right)\left(C_{\mathrm{g}} / C_{\mathrm{s}}{ }^{0}\right)
$$

The elution volume $V_{\mathrm{e}}$ of an ionic solute in GPC is given by

$$
V_{\mathrm{e}}=V_{0}+k \cdot V_{\mathrm{i}}
$$

where $V_{\mathrm{o}}$ and $V_{\mathrm{i}}$ are the void and inner volume of the column, respectively, and $k$ is the distribution coefficient of the solute considered. $V_{\mathrm{o}}$ can be determined by using a high molecular weight standard, and $V_{\mathrm{i}}$ by a low molecular weight standard. In terms of the definition of $k$, the distribution coefficient of the anion $k_{\mathrm{A}}$ and that of the cation $k_{\mathrm{K}}$ may be expressed as follows ${ }^{11}$ :

$$
k_{\mathrm{A}}=\left(C_{\mathrm{A}}^{\mathrm{i}} / C_{\mathrm{A}}{ }^{0}\right)
$$

and

$$
k_{\mathrm{K}}=\left(C_{\mathrm{K}}{ }^{\mathrm{i}} / C_{\mathrm{K}}{ }^{\circ}\right)
$$

Substitution of eq 10 in eq 7 leads to

$$
\left(k_{\mathrm{A}}\right)^{2}=-\left(k_{\mathrm{A}} / C_{\mathrm{s}}{ }^{\circ}\right)\left(C_{\mathrm{g}}\right)+R
$$

Similarly, a relation between $k_{\mathrm{K}}$ and $C_{\mathrm{s}}{ }^{\circ}$ is obtained as

$$
\left(k_{\mathrm{K}}\right)^{2}=\left(k_{\mathrm{K}} / C_{\mathrm{s}}{ }^{0}\right)\left(C_{\mathrm{g}}\right)+R
$$


If the elution behavior of the salt is governed by Donnan salt exclusion operative between the ionic solute and the fixed charges of the gel, and further if $R$ remains constant in the range of the salt concentration studied, a plot of $\left(k_{\mathrm{A}}\right)^{2}$ against $\left(k_{\mathrm{A}} / C_{\mathrm{s}}{ }^{0}\right)$ or $\left(k_{\mathrm{K}}\right)^{2}$ against $\left(k_{\mathrm{K}} / C_{\mathrm{s}}{ }^{\circ}\right)$ should give a straight line having a slope equal to $C_{\mathrm{g}}$ and intersecting with the ordinate axis at $R$.

The extention of the theory to the other types of salts is straightforward. For example, in the case of $z: 1$ type salts, $K^{z+}\left(\mathrm{A}^{-}\right)_{z}$, the following relations can be obtained:

$$
\left(k_{\mathrm{A}}\right)^{z+1}=-\left(C_{\mathrm{g}} / C_{\mathrm{s}}^{\mathrm{o}} \cdot z\right)\left(k_{\mathrm{A}}\right)^{z}+R^{\prime}
$$

and

$$
\left(k_{\mathrm{K}} z\right)^{(1+(1 / z))}=\left(C_{\mathrm{g}} / C_{\mathrm{s}}{ }^{0} \cdot z\right)\left(k_{\mathrm{K}} z\right)^{1 / z}+R^{\prime}
$$

with

$$
R^{\prime}=\left(\gamma_{ \pm}^{\mathrm{o}} / \gamma_{ \pm}^{\mathrm{i}}\right)^{z+1}
$$

Here $z$ is the algebraic valency of the cation (cation to anion charge ratio $=z: 1$ ) and $k_{\mathrm{K}} z$ is the distribution coefficient of cation $K^{z+}$. The values of $k_{\mathrm{K}} z$ and $k_{\mathrm{A}}$ can be used to evaluate $R^{\prime}$ by the following relation:

$$
R^{\prime}=\left(k_{\mathrm{A}}\right)^{z}\left(k_{\mathrm{K}} z\right)
$$

\section{EXPERIMENTAL}

\section{Materials}

All chemicals were of chromatographic grade and used without further purification. Deionized water was used throughout the experiments. A Sephadex G-25 gel and a Blue Dextran $2000 \quad\left(M_{w}=2 \times 10^{6}\right)$ were purchased from Pharmacia Fine Chemicals Inc.

\section{Procedure}

A series of GPC experiments were carried out in a manner similar to that described by Sebille et al. ${ }^{9}$ The column used was a glass tube $(1.1 \times 50 \mathrm{~cm})$ packed with the Sephadex G-25 gel and thermostated at $25 \pm 0.5^{\circ} \mathrm{C}$. The volume of the injected water was always $1 \mathrm{ml}$. The elution was carried out by gravitational force at a flow rate of $120 \mathrm{ml} \mathrm{h}^{-1}$. The eluted liquid was admitted into a fraction collector on a weight basis to give fractions of $3.5 \mathrm{~g}$ each. The concentration of metal cations in each fraction was determined by a Shimadzu Model AA-
$610 \mathrm{~S}$ atomic absorption spectrometer. The concentration of chloride ions was determined by a Horiba F-7II pH-meter provided with a chloridespecific ion electrode (8002-05T, Horiba Instruments Inc., Kyoto) and a reference calomel electrode having a double salt bridge (Double Junction 2535A-057, Horiba Instruments Inc.). The $\mathrm{pH}$-meter had a sensitivity of $\pm 0.02 \mathrm{pH}$ unit.

The void volume of the column, $V_{0}$, was estimated from the peak position in the elution curve for the Blue Dextran 2000, while $\mathrm{HCl}$ was used for determination of the total liquid volume of the column, $\left(V_{0}+V_{\mathrm{i}}\right)$.

\section{Ion Exchange Capacity Determination on Sephadex $\mathrm{Gel}$}

The anionic charges incorporated in the Sephadex G-25 gel phase were determined as follows. The gel was converted to the $\mathrm{Na}^{+}$form by washing it successively with $0.1 \mathrm{~N} \mathrm{NaOH}, 0.1 \mathrm{M}$ $\mathrm{NaCl}$ and distilled water. The elution was then carried out with $0.001 \mathrm{~N} \mathrm{HCl}$ until no further $\mathrm{Na}^{+}$could be detected in the eluent, and the amount of $\mathrm{Na}^{+}$displaced by $\mathrm{H}^{+}$was determined from the area under the elution profile of $\mathrm{Na}^{+}$.

\section{RESULTS AND DISCUSSION}

\section{Concentration of Fixed Anionic Charges}

The cation exchange capacity of a gel having fixed anionic charges may be determined by converting the gel to the $\mathrm{Na}^{+}$form and then measuring the amount of $\mathrm{Na}^{+}$displaced by the acid. Figure 1 shows the elution profile of $\mathrm{Na}^{+}$eluted from a column packed with the Sephadex G-25 gel in the $\mathrm{Na}^{+}$form. The elution was carried out with $0.001 \mathrm{~N}$

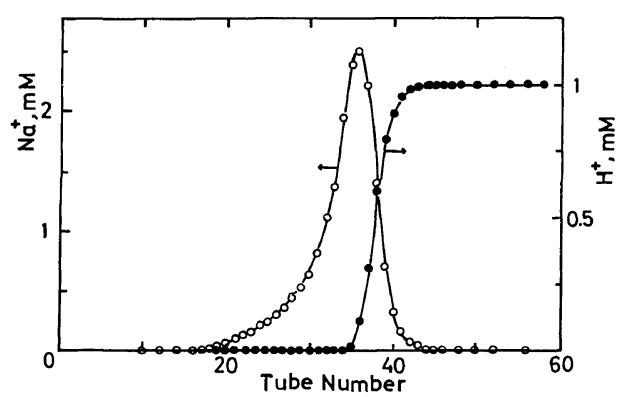

Figure 1. Elution profiles of $\mathrm{Na}^{+}$and $\mathrm{H}^{+}$from a Sephadex G-25 column in the $\mathrm{Na}^{+}$form when the gel column was washed with $0.001 \mathrm{~N} \mathrm{HCl}$. 
$\mathrm{HCl}$. Figure 1 also shows the change in $\mathrm{pH}$ of the eluent. The area under the $\mathrm{Na}^{+}$elution profile gives the value of the cation exchange capacity of the gel. In the present case, the value of $2.2 \pm 0.2 \mu$ eq. $\mathrm{g}^{-1}$ dry gel was obtained. The concentration of fixed anionic charges in the gel phase, $C_{\mathrm{g}}$, was calculated from the value of $2.2 \mu$ eq. $\mathrm{g}^{-1}$ for the cation exchange capacity and the inner volume of the column used in this study, assuming that the anionic charges are uniformly distributed in the gel. The value of $C_{\mathrm{g}}$ thus found was $9.5 \pm 1.0 \times 10^{-4} \mathrm{~mol} \mathrm{l}^{-1}$.

\section{Chromatograms by Conventional GPC Procedure}

Figure 2 shows chromatograms of $\mathrm{NaCl}$ eluted with pure water as a function of the sample concentration. The elution was carried out on a Sephadex G-25 column at $25^{\circ} \mathrm{C}$ and the gel was in the $\mathrm{Na}^{+}$form. The chromatograms for $\mathrm{NaCl}$ were obtained by measuring the concentration of $\mathrm{Na}^{+}$in the eluent. It can be seen that the elution volume at the peak position, $V_{\mathrm{e}}{ }^{*}$, and the shape of the curve strongly depend on the sample concentration injected, while the incipient rise of each chromatogram appears at the position close to the void volume, which is independent of the sample concentration. This elution behavior of $\mathrm{NaCl}$ agrees well with that reported by Neddermeyer et al. ${ }^{1}$ and Rochas et al. ${ }^{4}$ Such variation in $V_{\mathrm{e}}^{*}$ with the sample concentration as observed here has been qualitatively attributed to the Donnan salt exclusion effect, ${ }^{11}$ but, as may be recognized from the chromatograms in Figure 2, it does not seem that any unique relation between $V_{\mathrm{e}}{ }^{*}$ and the sample concentration in the column can be found. In addition, the sample concentration in the column is no longer the same as that injected, because of the sample band broading. Thus it is obvious that no quantitative analysis of the salt exclusion effect can be made using the chromatograms obtained by the conventional GPC procedure.

\section{Chromatograms by Equilibrium Saturation Method}

Figure 3 shows chromatograms for $\mathrm{NaCl}$ obtained by the equilibrium saturation method as a function of the sample concentration. The experiments were carried out as follows. At first, a Sephadex G-25 column was equilibrated with an aqueous solution containing only $\mathrm{NaCl}$ of known concentration, and then a small amount of pure

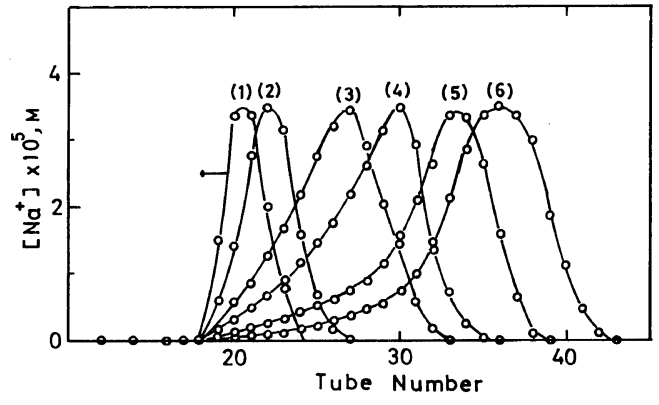

Figure 2. Chromatograms for various concentrations of $\mathrm{NaCl}$ eluted with pure water from a Sephadex G-25 column. The heights of chromatograms are reduced except for (1). Concentration: (1), $5 \times 10^{-4} \mathrm{M}$; (2), $10^{-3} \mathrm{M}$; (3), $5 \times 10^{-3} \mathrm{M}$; (4), $10^{-2} \mathrm{M}$; (5), $2.5 \times 10^{-2} \mathrm{M}$; (6), $10^{-1} \mathrm{M}$.

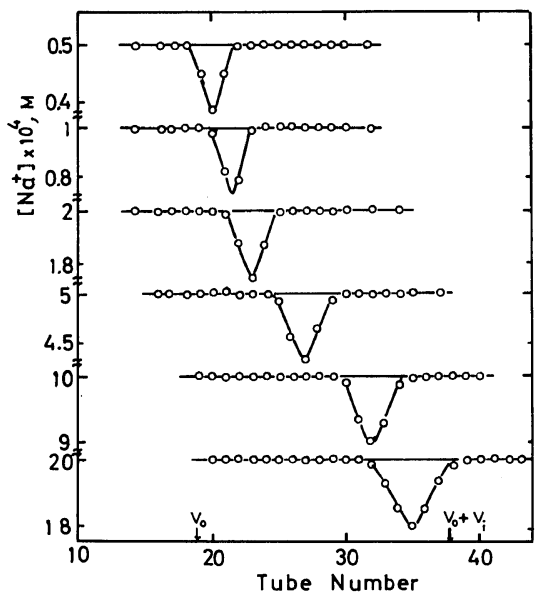

Figure 3. Chromatograms for various concentrations of $\mathrm{NaCl}$ obtained by the equilibrium saturation method on a Sephadex G-25 column.

water was injected into the column and eluted with the same salt solution as used for the equilibration. It can be seen from Figure 3 that one negative peak, which reflects the deficiency of $\mathrm{NaCl}$ caused by the injection of pure water, appeared between $V_{0}$ and $\left(V_{0}+V_{\mathrm{i}}\right)$. The base-line concentration corresponds to the concentration of $\mathrm{NaCl}$ in the outside of the gel, $C_{\mathrm{s}}{ }^{\circ}$. Here it should be emphasized that all the chromatograms were symmetrical. Similar chromatograms were also obtained for $\mathrm{CaCl}_{2}$ and $\mathrm{AlCl}_{3}$. The dependence of $V_{\mathrm{e}}{ }^{*}$ upon $C_{\mathrm{s}}{ }^{\circ}$ for these sample salts is shown in Figure 4. It can be seen that $V_{\mathrm{e}}^{*}$ for each salt decreases with increasing salt concen- 


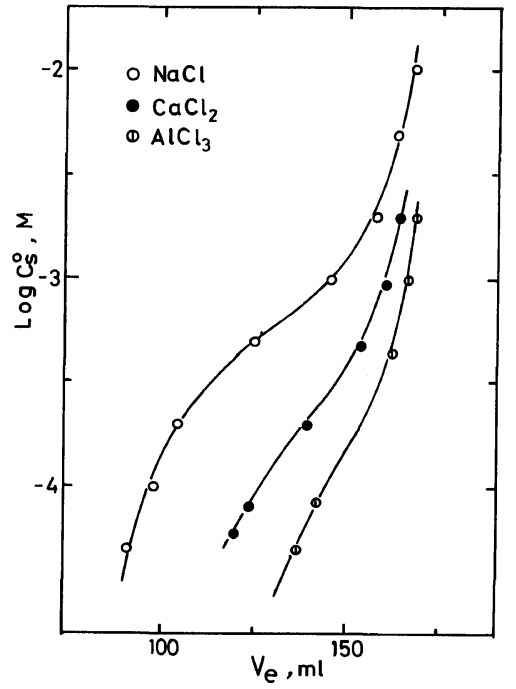

Figure 4. Dependence of the elution volume for various salts on the salt concentration in the eluent: $O$, $\mathrm{NaCl} ; \bigcirc, \mathrm{CaCl}_{2} ;$ (1), $\mathrm{AlCl}_{3}$.

tration. The results also indicate that $V_{\mathrm{e}}{ }^{*}$ at a fixed concentration becomes larger in the order of $\mathrm{AlCl}_{3}$, $\mathrm{CaCl}_{2}, \mathrm{NaCl}$. In other words, $V_{\mathrm{e}}{ }^{*}$ at the same salt concentration increases with the valency of salt cation.

In connection with Figure 3, it should be noted that the chromatograms obtained correspond not to the elution behavior of $\mathrm{Na}^{+}$injected but to that of co-ion $\mathrm{Cl}^{-}$, although these chromatograms were obtained by measuring the concentration of $\mathrm{Na}^{+}$in the eluent. The reason for this is that some of the $\mathrm{Na}^{+}$ions may be exchanged for those attached to the gel because of the cation exchangeability of the gel. This is the mechanism govering ion-exchange chromatography. The elution volume for each negative peak in Figure 3 allows us to evaluate the distribution coefficient of $\mathrm{Cl}^{-}$ion, $k_{\mathrm{Cl}^{-}}$, according to eq 9 .

The distribution coefficient of the salt cation, $k_{\mathrm{K}}$, will require additional experimentation for its estimation. Generally, $k_{\mathrm{K}}$ is difficult to estimate. In the case of $\mathrm{Na}^{+}$ions, it was possible to estimate $k_{\mathrm{Na}^{+}}$ from the following simple experiments. After the column had been equilibrated with an aqueous solution containing $\mathrm{NaCl}$ of known concentration, a small volume of $\mathrm{KCl}$ solution with a concentration exactly the same as that of $\mathrm{NaCl}$ was injected into the column, and eluted with the same

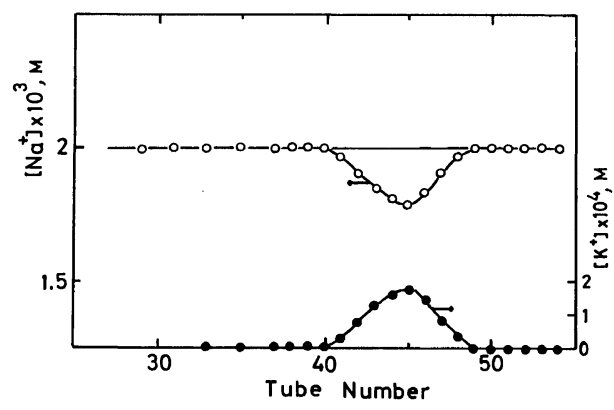

Figure 5. Chromatogram of $\mathrm{KCl}$ eluted with the same concentration of $\mathrm{NaCl}$ as that of injected $\mathrm{KCl}$. Sample, $2 \times 10^{-3} \mathrm{M} \mathrm{KCl}$ (in water). Eluent, $2 \times 10^{-3} \mathrm{M}$ $\mathrm{NaCl}$. For details, see text.

$\mathrm{NaCl}$ solution used for the equilibration. A negative peak related to the $\mathrm{Na}^{+}$concentration and a positive peak to the $\mathrm{K}^{+}$concentration appeared in the chromatogram. Figure 5 shows a typical chromatogram obtained for the $\mathrm{NaCl}-\mathrm{KCl}$ system on a Sephadex G-25 column. The total concentration of both cations $\mathrm{Na}^{+}$and $\mathrm{K}^{+}$and the concentration of co-ion $\mathrm{Cl}^{-}$were kept constant, as can be seen from the chromatograms. The same result was also obtained by an inverse procedure in which a $\mathrm{NaCl}$ solution was injected and eluted with a $\mathrm{KCl}$ solution. These results indicate that the elution volume, i.e., the distribution coefficient of $\mathrm{Na}^{+}, k_{\mathrm{Na}^{+}}$, is almost the same as that of $\mathrm{K}^{+}, k_{\mathrm{K}^{+}}$. Thus, the values of $k_{\mathrm{Na}^{+}}$or $k_{\mathrm{K}}$ + may be estimated using either one of the procedures mentioned above. In the present study, the values of $k_{\mathrm{Na}^{+}}$were determined by the former procedure. However, no suitable method for the estimation of the distribution coefficients of other types of cations such as $\mathrm{Ca}^{2+}$ and $\mathrm{Al}^{3+}$ is available at the present.

\section{Comparison with Theory}

Figure 6 shows plots of $\left(k_{\mathrm{Cl}^{-}}\right)^{z+1}$ against $\left(k_{\mathrm{Cl}^{-}}\right)^{z} /$ $\mathrm{C}_{\mathrm{s}}{ }^{\mathrm{o}} \cdot z$. The data points fall approximately on a common single line, which is essentially linear throughout the entire range of concentration $C_{\mathrm{s}}{ }^{\circ}$ studied. This suggests that the observed salt exclusion may be attributed to the Donnan salt exclusion effect due to the fixed charges of the gel. With the aid of eq 14, the value of $C_{\mathrm{g}}$ was determined from the slope of the straight line to be $9.0 \times 10^{-4} \mathrm{~mol}^{-1}$. This $C_{\mathrm{g}}$ value is in good agreement with that obtained from the direct measure- 


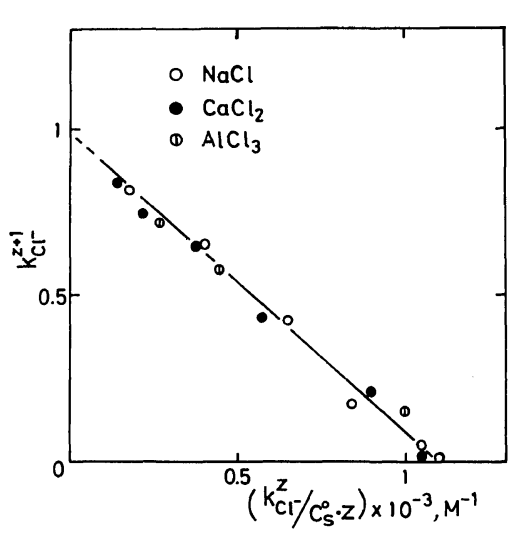

Figure 6. Plot of eq 14 with the same data as shown in Figure 4.

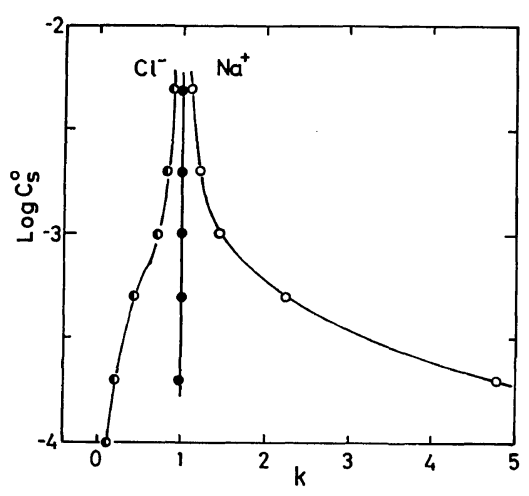

Figure 7. Relation between the distribution coefficient and salt concentration. The products of $k_{\mathrm{Na}^{+}}$and $k_{\mathrm{Cl}^{-}}$ are also plotted by filled circles: $\bigcirc, \mathrm{Na}^{+} ; \mathbf{D}, \mathrm{Cl}^{-}$.

ment of the ion exchange capacity of the gel.

The value of $R^{\prime}$ in eq. 14 was found to be close to unity. This indicates that the mean molar ionic activity coefficient of the salt is equal in both phases, i.e., the outside and inside of the gel. Strictly speaking, $R^{\prime}$ should be a function of the salt concentration. However, our results imply that the concentration dependence of $R^{\prime}$ is negligible for the range of low salt concentration studied here.

Next, the elution behavior of the salt cation, $\mathrm{Na}^{+}$ ion was considered. Figure 7 shows the dependence of $k_{\mathrm{Na}^{+}}$upon $C_{\mathrm{s}}{ }^{\circ}$. The same plots for $k_{\mathrm{Cl}^{-}}$are also shown for comparison. It can be seen that, in contrast to $k_{\mathrm{Cl}^{-}}$, the value of $k_{\mathrm{Na}}$ increases with decreasing salt concentration. It is clear that this result fulfils the requirement of eq 15. Furthermore, to test the validity of eq 17 , the products of $k_{\mathrm{Na}^{+}}$and $k_{\mathrm{Cl}^{-}}$, i.e., $R^{\prime}$, are shown by filled circles in Figure 7, which indicates that $R^{\prime}$ is approximately constant and equal to unity.

From these results it may be concluded that the anomalous elution behavior of simple salts can be quantitatively interpreted in terms of the Donnan equilibrium established between the outside and inside of the gel carrying fixed charges.

\section{REFERENCES}

1. P. A. Neddermeyer and L. B. Rogers, Anal. Chem., 40, 755 (1968).

2. P. A. Neddermeyer and L. B. Rogers, Anal. Chem., 41, 94 (1969).

3. N. Yoza, J. Chromatogr., 86, 325 (1973).

4. C. Rochas, A. Domard, and M. Rinaudo, Eur. Polym. J., 16, 135 (1980).

5. M. Rinaudo and J. Desbrieres, Eur. Polym. J., 16, 849 (1980).

6. R. Domard, M. Linaudo, and C. Rochas, J. Polym. Sci., Polym. Phys. Ed., 17, 673 (1979).

7. A. R. Cooper and D. P. Matzinger, J. Appl. Polym. Sci., 23, 419 (1979).

8. T. Lindström, A. d. Ruvo, and C. Soremark, J. Polym. Sci., Polym. Chem. Ed., 15, 2029 (1977).

9. B. Sebille, N. Thuaud, and J. P. Tillement, J. Chromatogr., 180, 103 (1979).

10. E. Glueckauf and R. E. Watts, Proc. R. Soc. London, Ser. A, 268, 339 (1979).

11. R. L. Pecsok and D. Sauders, Sep. Sci., 3, 325 (1968). 